\title{
Topological charge pumping in the interacting bosonic Rice-Mele model
}

\author{
A. Hayward, ${ }^{1,2,3}$ C. Schweizer, ${ }^{3,4}$ M. Lohse, ${ }^{3,4}$ M. Aidelsburger, ${ }^{3,4}$ and F. Heidrich-Meisner ${ }^{1}$ \\ ${ }^{1}$ Institute for Theoretical Physics, Georg-August-Universität Göttingen, Friedrich-Hund-Platz 1, 37077 Göttingen, Germany \\ ${ }^{2}$ Arnold Sommerfeld Center for Theoretical Physics, Ludwig-Maximilians-Universität München, 80333 München, Germany \\ ${ }^{3}$ Fakultät für Physik, Ludwig-Maximilians-Universität, Schellingstrasse 4, 80799 München, Germany \\ ${ }^{4}$ Max-Planck-Institut für Quantenoptik, Hans-Kopfermann-Strasse 1, 85748 Garching, Germany
}

(Received 17 October 2018; revised manuscript received 30 November 2018; published 27 December 2018)

\begin{abstract}
We investigate topological charge pumping in a system of interacting bosons in the tight-binding limit, described by the Rice-Mele model. An appropriate topological invariant for the many-body case is the change of polarization per pump cycle, which we compute for various interaction strengths from infinite-size matrixproduct-state simulations. We verify that the charge pumping remains quantized as long as the pump cycle avoids the superfluid phase. In the limit of hardcore bosons, the quantized pumped charge can be understood from single-particle properties such as the integrated Berry curvature constructed from Bloch states, while this picture breaks down at finite interaction strengths. These two properties-robust quantized charge transport in an interacting system of bosons and the breakdown of a single-particle invariant-could both be measured with ultracold quantum gases extending a previous experiment [Lohse et al., Nat. Phys. 12, 350 (2016)]. Furthermore, we investigate the entanglement spectrum of the Rice-Mele model and argue that the quantized charge pumping is encoded in a winding of the spectral flow in the entanglement spectrum over a pump cycle.
\end{abstract}

DOI: 10.1103/PhysRevB.98.245148

\section{INTRODUCTION}

The experimental and theoretical investigation of topological states of matter is a key topic in condensed matter physics [1-3], ultracold quantum gases [4-6], and photonics $[7,8]$. While the theoretical classification of noninteracting symmetry-protected topological states is complete $[9,10]$, the investigation of topology beyond noninteracting particles, zero temperature, and closed quantum systems is an active field of research. In recent years, different characterizations have been developed, showing that only certain aspects of topology can survive in mixed states (see, e.g., Refs. [11-16]) or nonequilibrium situations [17-23]. However, for interacting systems at zero temperature, generalizations of topological invariants have been identified [11,14,24-30], and it was shown that for many examples, such as Chern insulators, the topology is preserved for sufficiently small interactions (see, e.g., Refs. [26,31-35]).

Experiments with ultracold atomic gases are now able to explore aspects of topological physics providing direct access to nontrivial quantities such as Chern numbers [36-39], Berry phases, Berry curvatures [39-42], the dynamics of edge states [43-45], or the topological properties of one-dimensional (1D) symmetry-protected topological phases such as the SuSchrieffer-Heeger model (SSH) [46-48]. Moreover, some of the most paradigmatic two-dimensional (2D) lattice models

Published by the American Physical Society under the terms of the Creative Commons Attribution 4.0 International license. Further distribution of this work must maintain attribution to the author(s) and the published article's title, journal citation, and DOI. with topological band structures were implemented, including the Hofstadter model [49-51] and variants [43-45,52,53] and the Haldane model $[38,40]$. Most of these experiments, however, focused on noninteracting systems and there is a strong ambition to go beyond single-particle dynamics and to investigate topological phenomena in genuine many-body systems.

Here, we consider the effects of interactions in a topological charge-pumping setup that is realizable in quantum-gas experiments. Topological charge pumps were introduced in the seminal work by Thouless [54]. In the noninteracting case, it is well known that an adiabatic cyclic evolution in the space of Hamiltonian parameters leads to a quantized charge transport per cycle. In fact, this quantization is intimately related to the quantized Hall conductivity of a (2D) Chern insulator, in the sense that the cyclic parameter of the 1D Hamiltonian (that describes the charge pump) corresponds to a quasimomentum in the 2D model [32,54]. Experiments on topological Thouless pumping were performed with ultracold atoms [55-58] and photons [59,60] and have recently been extended to higher dimensions [61,62].

Interacting charge pumps have been studied theoretically, both for fermions [63,64] and bosons [65-67]. Originally, it was shown by Thouless that quantization is unaffected by weak interactions under fairly broad assumptions [32]. However, numerical simulations have shown that strong interactions can lead to a breakdown of the quantized pumping by closing the many-body gap [64]. Moreover, charge pumps are convenient tools to characterize the topology of interacting many-body systems in numerical simulations (see, e.g., Refs. [68-70]).

In the limit of hardcore bosons, the interacting 1D charge pump, as reported in the experimental work Ref. [55], has a 
simple interpretation. The model can be mapped onto noninteracting spinless fermions. For a completely filled band of fermions, all quasimomentum components are homogeneously populated and the total amount of charge transported per cycle is determined by the sum of the Berry curvature over all quasimomenta. In this paper, we are interested in the regime of finite interaction strengths, where this mapping is no longer valid. Finite interaction strengths result in an inhomogeneous momentum distribution and a many-body characterization of the transport via the polarization needs to be used.

Let us give a more formal account of the relevant topological invariants. To quantify a charge pump, one can consider the evolution of the many-body polarization $P(t)$ of a state $|\Psi(t)\rangle$ over the course of the adiabatic driving of a timedependent Hamiltonian $H(t)$.

The modern theory of polarization associates $P$ with the mean position of the charge distribution per unit cell in the case of translationally invariant states [71]. For a system of length $L a$ with periodic boundary conditions, one can define the polarization for a many-body wave function $|\Psi(t)\rangle$ via [72]

$$
P(t)=\frac{q a}{2 \pi} \operatorname{Im} \ln \left\langle\Psi(t)\left|e^{\frac{i 2 \pi}{L a} \hat{X}}\right| \Psi(t)\right\rangle \quad(\bmod q a),
$$

where $\hat{X}=\sum_{i}^{\mathrm{B} \times \mathrm{L}} x_{i} \hat{n}_{i}$ is the position operator, $\hat{n}_{i}$ and $x_{i}$ are, respectively, the density operator and position of site $i, q$ is the charge per particle, $a$ is the length of the unit cell, $L$ is the number of unit cells, and $B$ is the number of sites per unit cell. Importantly, $P$ is only defined modulo $q a$ and it has the units of a dipole moment. We keep $q$ in the equations for clarity, yet for our case of neutral atoms, $q=1$.

In general, the total transported charge $\Delta Q\left(t_{1} ; t_{2}\right)$ between times $t_{1}$ and $t_{2}$ can be related to the polarization via

$$
\Delta Q=\int_{t_{1}}^{t_{2}} d t J(t)=\frac{1}{a} \int_{t_{1}}^{t_{2}} d t \partial_{t} P(t),
$$

where $J(t)$ is the current density of the system.

Now we consider the case of charge pumps which are described by time-periodic Hamiltonians, such that $H(t+$ $T)=H(t)$, with $T$ the period of the pump cycle. Assuming perfect adiabaticity, the polarization is cyclic in $T$ as well, with $P(T) \bmod q a=P(0)$. The final expression in Eq. (2) with $\Delta t=T$ is the winding number of the polarization, which implies the quantization of the pumped charge for each cycle.

The expression for the many-body polarization reduces to the usual form for noninteracting fermions for a filled band $[71,73,74]$ :

$$
P_{\mathrm{NI}}(t)=\frac{-i q}{2 \pi} \int_{-\pi / a}^{\pi / a} d k\left\langle u(k, t)\left|\partial_{k}\right| u(k, t)\right\rangle,
$$

where $|u(k, t)\rangle$ are single-particle momentum-eigenstates of the system and $k \in[-\pi / a, \pi / a)$ is the quasimomentum. In this case, the polarization is proportional to the Zak phase associated with a filled band, which is defined modulo $2 \pi$ [75]. The charge pumped per cycle $(\Delta Q)$ can then be expressed as

$$
\Delta Q=\frac{q}{2 \pi} \int_{-\pi / a}^{\pi / a} d k \int_{0}^{T} d t \Omega(t, k)=q v,
$$
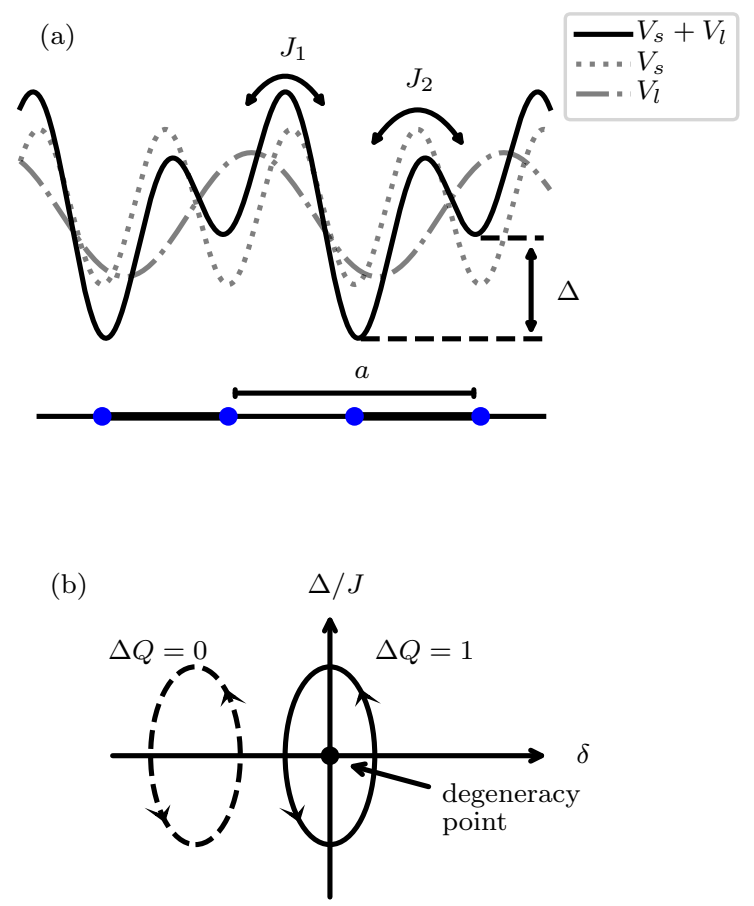

FIG. 1. Schematic of the Rice-Mele model and of exemplary pump cycles: (a) A bichromatic optical potential created from two optical standing waves with wavelength $\lambda_{l}=2 \lambda_{s}$ can be described by a tight-binding lattice model, the Rice-Mele model, with staggered potential $\Delta$ and alternating tunneling strength $J_{i}=J(1+$ $\left.(-1)^{i} \delta\right)$, where $i=\{1,2\} . \delta$ is the dimensionless dimerization parameter [Eq. (7)] and $a=\lambda_{l} / 2$ is the length of the unit cell. (b) These parameters can be tuned by changing the lattice potentials $V_{l, s}$, and the relative phase $\phi$ of the two optical potentials [Eq. (6)]. Periodic tuning of these parameters defines a pump path. The pumped charge $\Delta Q$ of a pump path can be either $\Delta Q=1$ (solid line) or $\Delta Q=0$ (dashed line) depending on whether the pump cycle encloses the degeneracy point at $\delta=\Delta=0$ or not.

where $v$ is the Chern number associated with the Berry curvature of the pump cycle:

$$
\Omega(k, t)=i\left\langle\partial_{k} u(k, t) \mid \partial_{t} u(k, t)\right\rangle-c . c .,
$$

defined on a torus of dimension $T \times 2 \pi / a$. If Eq. (4), with a suitable choice of states $|u(k, t)\rangle$, yields the actual quantized charge in a many-body system, then we say that a singleparticle description applies.

In this paper, we investigate interacting bosonic charge pumps in the tight-binding Rice-Mele model [76], which consists of alternating hopping-matrix elements and alternating onsite potentials [see Fig. 1(a)]. To model a charge pump, both parameters are varied periodically [see Fig. 1(b)]. We pursue two main goals: First, we reveal the breakdown of the single-particle interpretation in a parameter regime, where the charge pump remains topologically protected away from the limit of hardcore bosons. Measuring both the quasimomentum distribution function and the center-of-mass motion of the cloud as a measure of the quantization of charge pumping are sufficient to demonstrate this breakdown in an experiment. Both observables are readily available in existing cold-atom experiments. Realizing this regime would 
constitute a nontrivial way of observing topologically protected charge pumping in an interacting system and may be an important step toward experimental studies of topological effects in many-body systems. Second, we investigate entanglement properties and observe that the quantized charge pump is reflected in a winding of the spectral flow in the entanglement spectrum.

We use matrix-product-state (MPS) methods in an infinitesystem size formulation (iDMRG) [77-79] to establish that the interacting topological charge pump remains quantized as long as the many-body gap does not close along the cycle for sufficiently strongly interacting bosons. As the system enters the superfluid region, correlations in the system diverge, and the transported charge is no longer quantized. Therefore, we focus on the Mott-insulating phase, where we explicitly demonstrate that there exists a parameter regime, in which an interpretation in terms of single-particle states results in a nonquantized transport, while the true transported charge actually remains protected and quantized.

We further relate the quantized charge pumping to the entanglement spectrum of the reduced density matrix in spatial bipartitions. The relationship between the entanglement spectrum, edge modes, and topological properties is well documented (see, e.g., Refs. [80-83]). In particular, Li and Haldane were able to show the correspondence between the low-energy structure of the entanglement spectrum of fractional quantum Hall states and their topology [80]. In 1D systems, symmetry-protected topological states $[84,85]$ imply an exact degeneracy in the entanglement spectrum [86]. Indeed, we show that charge pumping is encoded in a parity protection which appears for strong interactions. This results in a winding of the spectral flow over the pump cycle.

The paper is organized as follows. We introduce the interacting bosonic Rice-Mele model and its phase diagram in Sec. II, where we also present the infinite-system size density matrix renormalization group (iDMRG) method. In Sec. III, we introduce the pump cycles studied in this paper and show how charge pumping remains quantized in the presence of interactions via the winding of polarization. In Sec. IV, we discuss the one-body correlation functions and their associated quasimomentum distributions as possible experimental observables, and demonstrate the breakdown of a single-particle interpretation of quantized charge pumping away from the limit of hardcore bosons. Aspects relevant for experimental realizations are discussed in Sec. V. In Sec. VI, we relate the quantized pumping to the spectral flow in the entanglement spectrum. We conclude with a summary and an outlook in Sec. VII.

\section{INTERACTING RICE-MELE MODEL}

\section{A. Realization and Hamiltonian}

The Rice-Mele model [76] is a 1D tight-binding lattice model with alternating nearest-neighbor hopping-matrix elements $J_{i}=J\left(1+(-1)^{i} \delta\right), \delta \in[-1,1]$, and a staggered onsite potential with strength $\Delta$. In typical cold-atom experiments $[55,56]$, it can be realized with a bichromatic optical lattice, which consists of two optical lattices with wavelength $\lambda_{s}$ and $\lambda_{l}=2 \lambda_{s}$ as illustrated in Fig. 1(a). The corresponding optical potential is of the form

$$
V(x)=V_{l} \sin ^{2}\left(\frac{\pi x}{\lambda_{l}}-\frac{\phi}{2}\right)+V_{s} \sin ^{2}\left(\frac{\pi x}{\lambda_{s}}+\frac{\pi}{2}\right),
$$

where $V_{s, l}$ are the respective potential depths and $\phi$ is the relative phase between the two potentials. For sufficiently deep lattices, the physics can be described by the Rice-Mele tight-binding lattice model, which for bosonic atoms with local onsite interactions $U$ can be written as

$$
\begin{aligned}
H= & \sum_{i}\left[-J\left(1+(-1)^{i} \delta\right) \hat{a}_{i}^{\dagger} \hat{a}_{i+1}+H . c .\right. \\
& \left.+(-1)^{i} \frac{\Delta}{2} \hat{n}_{i}+\frac{U}{2} \hat{n}_{i}\left(\hat{n}_{i}-1\right)\right] .
\end{aligned}
$$

The operator $\hat{a}_{i}^{\dagger}$ creates a boson on site $i$ and $\hat{n}_{i}=\hat{a}_{i}^{\dagger} \hat{a}_{i}$. Conveniently, the model can be expressed in the dimensionless quantities $U / J, \delta$ and $\Delta / J$. In the following sections, we study pump cycles which are closed cycles in the $\delta$ and $\Delta / J$ parameter space, illustrated in Fig. 1(b).

In the absence of interactions $U=0$, the Rice-Mele Hamiltonian is a two-band model with the dispersion relation

$$
\epsilon_{k}= \pm J \sqrt{(\Delta / 2 J)^{2}+4\left[1-\left(1-\delta^{2}\right) \cos (k a)\right]},
$$

which has a degeneracy point at $\delta=\Delta=0$. This degeneracy determines the topology of the pump cycle. If the pump path encloses the degeneracy point, the corresponding pumped charge number is $\Delta Q=1$, while otherwise it is $\Delta Q=0$ [Fig. 1(b)].

\section{B. Phase diagram of the interacting Rice-Mele model}

\section{Hardcore-boson limit}

In the limit of hardcore interactions, $U / J=\infty$, and an average density per site of $\bar{n}=N /(2 L)=1 / 2$, with $N$ the number of bosons, the bosonic Rice-Mele model exhibits a Mott-insulating phase, except for $\delta=\Delta=0$. This can be understood by mapping the hardcore bosons to a system of free spinless fermions via the Jordan-Wigner transformation [87]. At $\bar{n}=1 / 2$, the fermions completely fill the lowest band, except at $\delta=\Delta=0$, where the system reduces to the uniform tight-binding chain with equal tunneling rates and the two bands merge into a single one. In the bosonic picture, this is the point where the system becomes superfluid. Away from $\bar{n}=1 / 2$, the system is always in a superfluid phase. Because of this, we will restrict our discussion to the average density $\bar{n}=1 / 2$ for the following sections.

\section{2. iDMRG calculations for finite $U / J$}

The 1D Bose-Hubbard model at integer filling has a gapped Mott-insulating state at large $U / J$ and a gapless superfluid state with algebraically decaying correlations for sufficiently low $U / J$ [88]. Similar physics applies to the bosonic interacting Rice-Mele model at filling $\bar{n}=1 / 2$ (see, for instance, Refs. $[89,90])$, which also exhibits a superfluid-toMott-insulator transition. A Mott insulator with a noninteger site occupancy is sometimes referred to as a fractional Mott insulator [91]. 


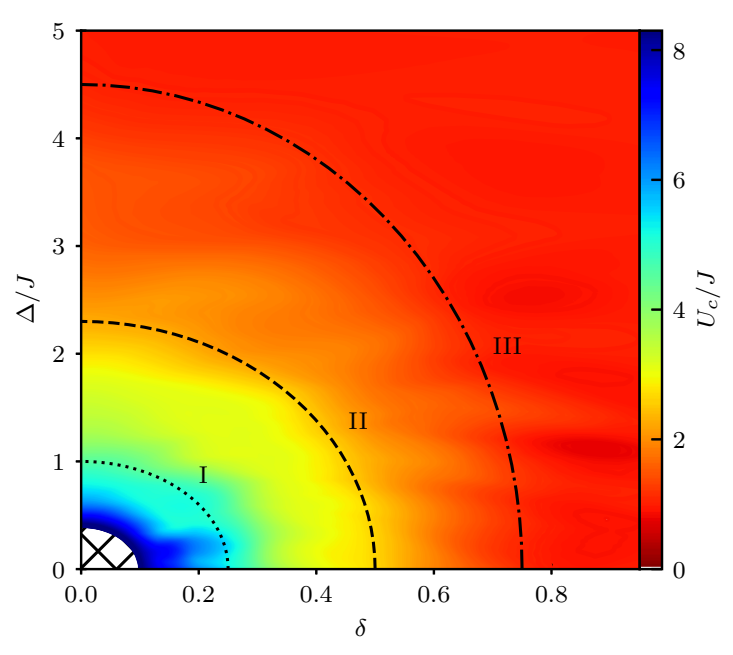

FIG. 2. Phase diagram of the interacting bosonic Rice-Mele model: Upper bound of the critical interaction strength $U_{c} / J$ as a function of the dimensionless parameters $\delta$ (dimerization in the hopping-matrix elements) and $\Delta / J$ (staggered potential). The hatched region close to the origin has not been computed. The lines show the paths of the pump cycles studied in this paper as defined in Eq. (10): dotted, path I: $R_{\delta}=0.25, R_{\Delta}=1.0$; dashed, path II: $R_{\delta}=0.5, R_{\Delta}=2.3$; dash-dotted, path III: $R_{\delta}=0.75, R_{\Delta}=4.5$.

To study the phase diagram of the interacting Rice-Mele model, we use iDMRG simulations [77] and compute infinitesystem-size matrix-product-states (iMPS) approximating the ground state. This allows us to avoid edge effects and to treat the problem directly in the thermodynamic limit. For Hamiltonians with a gapped ground state, an iMPS approximates the ground state very accurately. However, as the correlation length diverges upon approaching a critical phase, the finite bond dimension used in the simulations imposes an effective length scale. To understand the physics independently of this effective length scale-this is especially important in the superfluid phase-we employ a scaling analysis in the bond dimension to elucidate the ground-state physics. All computations are performed with a $U(1)$ symmetry preserving code with a bond dimension $\chi$ of up to a maximum of $\chi=2000$ using the tensor library developed in Ref. [92]. Furthermore, the local bosonic basis must be truncated in DMRG calculations to a maximum number of bosons per site. We found that for all of the calculations in this paper, a maximum of six bosons per site is sufficient. For the parameters considered, we never find the occupation of the local state with six bosons to rise above $10^{-10}$.

In Fig. 2, we show a phase diagram for the bosonic Rice-Mele model. We obtain the critical interaction strength $U_{c} / J$ separating the superfluid $\left(U / J<U_{c} / J\right)$ from the Mott insulator $\left(U / J>U_{c} / J\right)$ as a function of the parameters $\delta$ and $\Delta$. This diagram was computed via a scaling analysis of the correlation length $\xi$ with respect to the onsite interaction strength. The superfluid-to-Mott-insulator transition at constant density is in the universality class of $O(d+1)$, with $d$ the dimension of the system. In our case with $d=1$, the transition is expected to be in the same class as the BerezinskiiKosterlitz-Thouless transition [93], which is the underlying assumption in our numerical analysis. Such transitions are notoriously difficult to pin down precisely, as correlations in the system scale as $\xi \propto \exp \left(\right.$ const $/ \sqrt{J / U_{c}-J / U}$ ) [94], and not as a power law like in higher dimensions. However, data from iDMRG calculations can provide an upper bound on $U_{c} / J$, which is sufficient for our purposes.

The correlation lengths are extracted by taking the secondlargest eigenvalue of the transfer matrix [95]. We converge the DMRG algorithm with a maximum bond dimension of $\chi=2000$. In practice, we enforce a discarded weight of $10^{-14}$ for each DMRG truncation step, which can be achieved with a bond dimension less than the maximum value. If the desired bound on the discarded weight cannot be achieved, we consider the state to be unconverged and discard this point. We find poor convergence of the DMRG calculations in the vicinity of the central degeneracy $(\Delta / J=\delta=0)$, as the correlation length here diverges even in the hardcore-boson limit. We therefore exclude a small area around the origin, indicated by the hatched region in Fig. 2. Note that results for the phase diagram along the special lines $\Delta=0$ [90] and $\delta=0$ [89] of the phase diagram were previously obtained and agree well with our own calculations.

For large but finite $U / J$, the system remains gapped. One can see this by finding the effective Hamiltonian in the singleoccupancy manifold. Tracing out multiple occupancies results in an effective interaction:

$$
H_{\mathrm{int}}^{\mathrm{eff}}=-\sum_{i} 4 J_{i}^{2}\left(\frac{1}{U+\Delta}+\frac{1}{U-\Delta}\right) \hat{n}_{i} \hat{n}_{i+1}+\mathcal{O}\left(\frac{J^{3}}{U^{2}}\right)
$$

Here, we interpret $\hat{n}_{i}=\hat{b}_{i}^{\dagger} \hat{b}_{i}$ as a hardcore-boson occupation operator [with $\left(b_{i}^{\dagger}\right)^{2}=0$ ] and $J_{i}=J\left(1+(-1)^{i} \delta\right)$ the alternating tunneling rate. We switch to the equivalent fermionic picture for an interpretation. The effective nearest-neighbor interaction leaves the Mott-insulating phase stable and does not induce a phase transition as long as the interaction strength is smaller than the band gap.

\section{CHARGE PUMPING AND MANY-BODY POLARIZATION}

\section{A. Pump cycles}

Topological charge pumping allows for a robust quantized transport of charge through adiabatic cyclic modulation of the system's parameters. In the studied Rice-Mele model, pumping can be induced by an adiabatic modulation of the dimerization $\delta$ and the onsite energy $\Delta / J$. However, it is essential that the origin of this $(\delta-\Delta / J)$ parameter space is enclosed by the pump path, as the degeneracy point at the origin is the source of the topological properties. In this paper, we focus on paths of the form

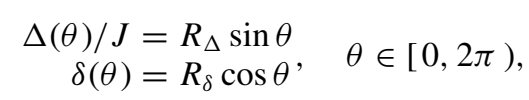

where we assume $J$ and $U$ to be constant throughout the pump cycle. A schematic of the path is shown in Fig. 3(a) while the actual paths used here are indicated in Fig. 2. We further define $\theta=2 \pi t / T$, assume adiabaticity and work in the instantaneous eigenbasis of $H(\theta)$. Hence, the charge transport becomes independent of the timescale $T$. 

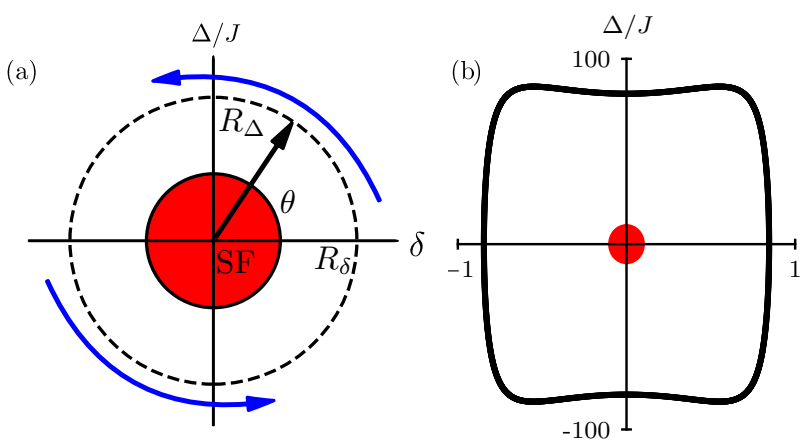

FIG. 3. Pump cycles studied in this work: (a) Parameter space of the interacting bosonic Rice-Mele model. At the center of the parameter space, there is a superfluid region (red), which shrinks as the interaction strength increases and becomes a point as $U / J \rightarrow \infty$. We consider a charge pump that traces a path through this parameter space and encircles the superfluid phase. The cycle is parameterized by the angle $\theta, R_{\delta}$, and $R_{\Delta}$. (b) Pump path used in the bosonic experiment [55] using the superlattice parameters $V_{s}=10 E_{r, s}, V_{l}=$ $20 E_{r, l}$ [Eq. (6)] where $E_{r,(l, s)}$ is the recoil energy of the respective lattice. The change in polarization associated with this path is shown in Fig. 4(b).

To make a connection to experiments, we compare our results to the pump path used in the bosonic experiment [55], where the charge pump was implemented with a bichromatic lattice as defined in Eq. (6). The pump cycle in the experiment, enclosing the degeneracy point $\delta=\Delta=0$, was realized by a variation of the relative phase $\phi$ at constant lattice depth and is depicted in Fig. 3(b). The quantized pump was executed in the strongly interacting regime $U \gg U_{c}$ and an average density $\bar{n} \simeq 1 / 2$.

\section{B. Charge pump in the limit of hardcore bosons}

In the limit of hardcore bosons, one can compute the polarization analytically from Eq. (3), using a mapping to free fermions via the Jordan-Wigner transformation. The lowest band cell-periodic wave function can be written as

$$
\left|u_{l}(k)\right\rangle=\frac{1}{\sqrt{2}}\left(\sqrt{1+\frac{\Delta}{2 \epsilon(k)}}, \sqrt{1-\frac{\Delta}{2 \epsilon(k)}} e^{-i \varphi(k)}\right),
$$

where $\varphi(k)=\mathcal{I m} \log \left[-(1+\delta)-(1-\delta) e^{i k a}\right]$. Substituting into Eq. (3) results in an expression for the polarization after some straightforward algebra:

$$
P(\delta, \beta)=a q \delta \beta \Pi\left(1-\delta^{2} \mid m\right),
$$

where $\beta=\Delta / \sqrt{16 J^{2}+\Delta^{2}}, \quad m=\left(1-\delta^{2}\right)\left(1-\beta^{2}\right)$, and $\left.\left.\Pi(w \mid z)=\int_{0}^{\pi / 2} d k\left(1-w \sin ^{2} k\right)\right)^{-1}\left(1-z \sin ^{2} k\right)\right)^{-1 / 2}$ is the complete elliptical integral of the third kind. This expression is valid for $\delta, \beta>0$, but can be related via symmetry to the rest of the parameter space.

The quantization of the pumped charge depends only on the topology of the pump path and the pumped charge is nonzero as long as the pump path encloses the degeneracy point. In the case where the path does not enclose the singularity, the polarization will have a winding number of zero, and hence no net transported charge $(\Delta Q=0)$. For example,

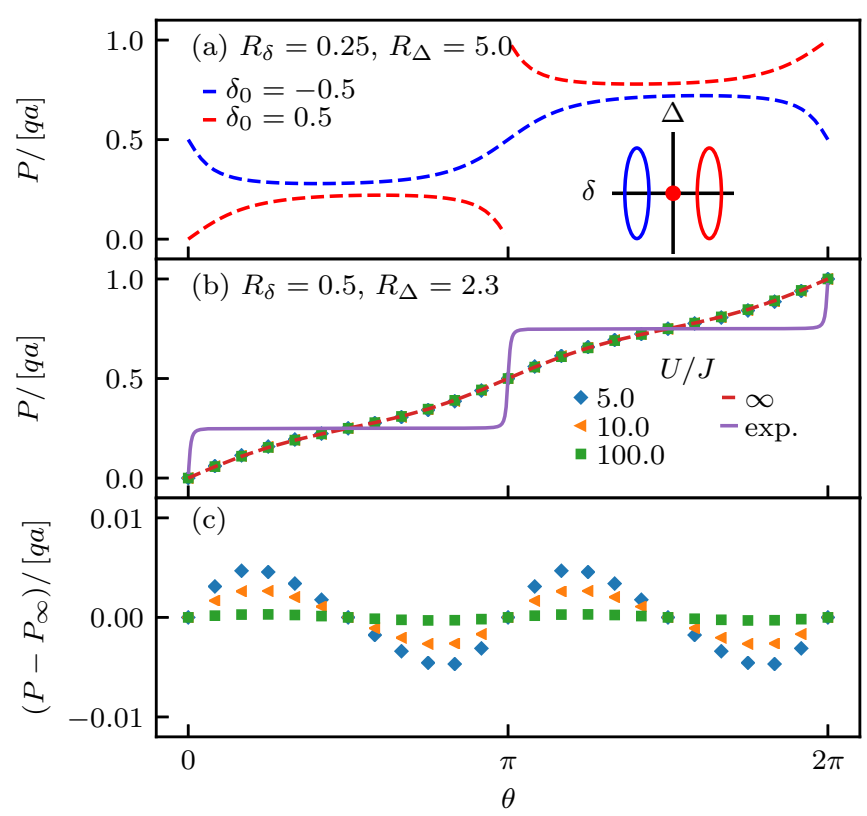

FIG. 4. Winding of the polarization: Polarization as determined by iDMRG computations [Eq. (16)] around the pump cycle with $R_{\delta}=0.5, R_{\Delta}=2.3$ and different values of the interaction strengths. The charge pumped over a cycle is the integrated slope of the polarization [Eq. (2)], which simply counts the winding of the polarization. (a) Polarization of hardcore bosons for paths which do not encircle the central degeneracy [see the inset in (a)], defined in Eq. (13). Notice that the polarization is cyclic, but has winding number zero, implying no pumped charge. (b) Polarizartion $P(\theta)$ for path II $\left(R_{\delta}=0.5, R_{\Delta}=2.3\right)$ for various interaction strengths $U / J=5,10,100, \infty$, and for the path used in the experiment (using $U / J=\infty$ ) [55]. (c) Polarization relative to the one in the hardcore limit, derived from the data in (b). $P_{\infty}$ is computed from the freefermion solution [Eq. (12)].

consider a path defined by

$$
\Delta(\theta) / J=R_{\Delta} \sin \theta, \quad \delta(\theta)=\delta_{0}+R_{\delta} \cos \theta,
$$

where $\delta_{0}$ is a constant shift to $\delta$. If $R_{\delta}<\left|\delta_{0}\right|$, then the path will not enclose the origin and the pump will transport no charge since, by Eq. (12), the polarization only oscillates around $P=$ 0 and does not wind at all, which is illustrated in Fig. 4(a). This becomes clear from Eq. (2) as the integral of the slope of the polarization is exactly zero for the path given in Eq. (13).

\section{Polarization from Schmidt values}

The MPS approximation to the ground state $|\Psi(\theta)\rangle$ can be factorized via a Schmidt decomposition (used in most MPS algorithms [79]) into the form

$$
|\Psi(\theta)\rangle=\sum_{\mu=0}^{\chi-1} \Lambda_{\mu}\left|\Psi_{\mu}^{L}(\theta)\right\rangle\left|\Psi_{\mu}^{R}(\theta)\right\rangle .
$$

The $\left|\Psi_{\mu}^{L(R)}(\theta)\right\rangle$ are states where $L$ and $R$ are the left and right semi-infinite partitions of the Rice-Mele chain, and the $\Lambda_{\mu}$ are the Schmidt values, which give the weight of each state.

For an infinite system, one can compute the polarization from the Schmidt values $\left(\Lambda_{\mu}\right)$. The Schmidt values are also 
the positive root of the eigenvalues of the reduced density matrix found by tracing out one half of the system:

$$
\rho_{L}=\operatorname{Tr}_{R}[\rho]=\sum_{\mu=0}^{\chi-1} \Lambda_{\mu}^{2}\left|\Psi_{\mu}^{L}(\theta)\right\rangle\left\langle\Psi_{\mu}^{L}(\theta)\right| .
$$

These numbers are directly accessible in any MPS code [79].

The $U(1)$ symmetry associated with charge conservation allows us to assign an integer quantum number $\Delta N_{\mu}$ to label each state $\left|\Psi_{\mu}^{L}\right\rangle$, which we refer to as the particle imbalance. These quantum numbers are associated with particle fluctuations across the cut in the system: The eigenstate of the reduced density matrix associated with $\Lambda_{\mu}$ corresponds to the state of the system with $\Delta N_{\mu}$ extra particles in the left-hand side of the system, and $-\Delta N_{\mu}$ in the right-hand side. Following the approach of Ref. [68], the polarization is then the expectation value of the particle imbalance:

$$
P=q a \sum_{\mu} \Delta N_{\mu} \Lambda_{\mu}^{2} .
$$

When working in the thermodynamic limit, the total number of particles in the system is not well defined. We therefore cannot ascribe an absolute value to the particle imbalances $\Delta N_{\mu}$. However, the $\Delta N_{\mu}$ are defined relative to each other such that $\Delta N_{\mu}-\Delta N_{\nu}$ is always the same for a pair $\mu, v$. This also implies that $P$ defined in Eq. (16) cannot be determined absolutely, but rather is only defined modulo $q a$, as for previous definitions of the polarization.

\section{Charge pumping for finite $U / J$}

The bosonic Rice-Mele charge pump requires a finite gap for adiabatic transport to take place. It is therefore necessary to avoid the superfluid regime along the path the pump cycle takes through parameter space.

In Fig. 4(b), we compare the pumped charge for a number of paths through parameter space at finite values of $U / J<\infty$ and compare to the hardcore-boson limit. As expected, the polarization rises monotonically with $\theta$. Surprisingly, the deviation $P-P_{\infty}$ [shown in Fig. 4(c)] of the polarization for finite $U / J$ from the $U / J \rightarrow \infty$ limit remains small, even as the correlation length in the system becomes large. These results show, from a full many-body calculation of the polarization from Eq. (1), that the pumped charge remains quantized away from the limit of hardcore bosons. This manifests itself in Fig. 4(c) as a vanishing difference between the polarizations of the finite and the infinite $U / J$ cases at $\theta=2 \pi$.

We would like to briefly comment on the consequence of bringing the pump through the superfluid region. Here, the particle-fluctuations across the system have a logarithmic divergence, with respect to the length of the system (or, in the case of iMPS with finite bond dimensions, with respect to the bond dimension). The relationship between particle fluctuations and entanglement entropy has been discussed extensively in Ref. [96], including the specific case of 1D systems. This divergence leads to an undefined polarization for an infinite system, as the particle imbalances $\Delta N_{\mu}$ in Eq. (16) inherit this divergence.

Similarly, the existence of gapless charge excitations means that there is, in general, no well-defined adiabatic limit, and any measured pumped charge will depend strongly on the details of the system and the speed with which the pump is executed. That is to say, there is no "universal" description of the pump in this regime.

\section{BREAKDOWN OF THE SINGLE-PARTICLE INTERPRETATION FOR $U / J<\infty$ IN THE MOTT-INSULATING PHASE}

In this section, we compute the quasimomentum distribution function of the bosons in the hardcore-boson limit as well as for finite $U / J$ in the Mott-insulating phase. We show that, unless very special pump cycles are chosen, the momentum distribution of the physical particles is never flat. Consequently, a single-particle picture, where the total transported charge results from the contribution of curvatures from each single-particle state, does not lead to a quantized value for the total transported charge.

Therefore, in general, the quantization of the pumped charge in a topological charge pumping experiment with bosons can only be established from many-body expressions using, e.g., the polarization [Eq. (1)]. We argue that the breakdown of a single-particle topological invariant could be demonstrated by measuring the quasimomentum distribution of each band and showing that the integral over this $n_{\alpha}(k, \theta)$ and the known single-particle Berry curvature is not quantized.

\section{A. Quasimomentum distribution function}

To compute the quasimomentum distribution function, we need to compute the one-particle density matrix (OPDM). It is straightforward to extract the OPDM from iMPS solutions using transfer-matrix methods [97].

The OPDM is given by $\rho_{i j}^{(1)}=\left\langle\Psi\left|\hat{a}_{i}^{\dagger} \hat{a}_{j}\right| \Psi\right\rangle$, where $|\Psi\rangle$ is, in our case, the many-body ground state. For a translationally invariant state, the corresponding OPDM $\rho^{(1)}$ will share the symmetry and can be block-diagonalized into blocks of dimensions $n \times n$, where $n$ is the size of the unit cell, and each block can be labeled by a quasimomentum.

The quasimomentum distribution usually measured in an experiment results from the projection of the OPDM onto the bands of a noninteracting model [88]. This corresponds to taking the diagonal elements of $\rho^{(1)}(k)$ when written in the eigenbasis of the noninteracting model. One can then associate a momentum distribution $n_{\alpha}(k, \theta)$ with each original band ( $\alpha=1, \ldots, n$ is the band index), as shown in Fig. 5 .

In the present case, we have a two-site unit cell, and we refer to the lower(upper) bands as $\alpha=l(u)$. In experiments, a flat $n_{\alpha}(k, \theta)$ is usually achieved by either using free fermions and choosing the filling appropriately or by localizing particles into individual sites. In the case of hardcore bosons, they, in general, already lack a perfectly flat momentum distribution for any filling of less than one particle per site and in equilibrium. Only after the relevant Jordan-Wigner transformation (see, e.g., Ref. [98]) the result is a flat quasimomentum distribution, but for spinless fermions. However, in a sufficiently deep lattice, the bosons will be largely localized, with an essentially flat distribution, which was exploited in Ref. [55] (also see our discussion in Sec. III B). 


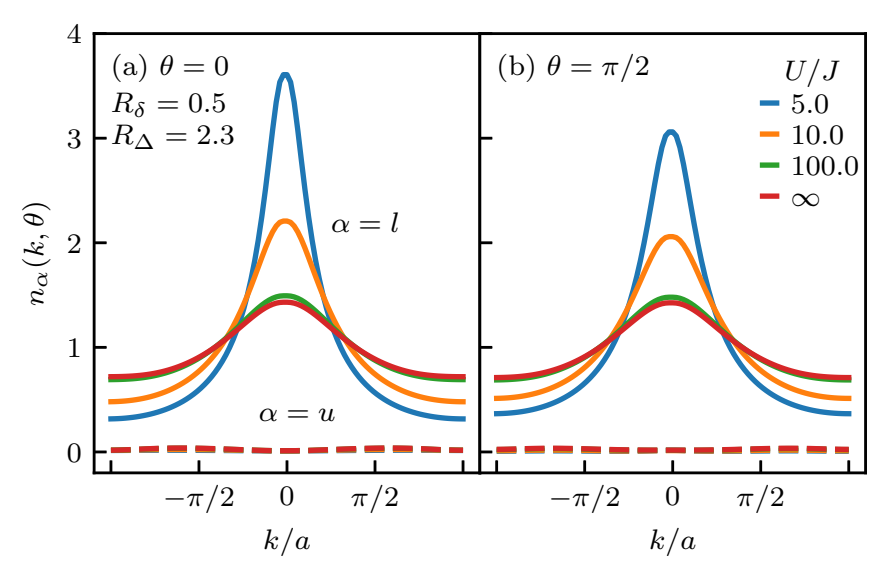

FIG. 5. Quasimomentum distribution of the interacting system: $n_{\alpha}(k, \theta)$ for the pump cycle with $R_{\delta}=0.5, R_{\Delta}=2.3$. Solid lines are the projection onto the lowest band $(\alpha=l)$ of the noninteracting Hamiltonian, the dashed lines are for the projection onto the upper band $(\alpha=u)$. (a) Distribution at $\theta=0$. (b) $\theta=\pi / 2$. For the path shown, the occupation density of the upper band is quite low: $n_{u}(k, \theta) \lesssim 0.03$ in the $U / J=\infty$ limit for all $\theta$. However, this occupation can be larger when the pump cycle is closer to the degeneracy. Note that for the experimental path of Ref. [55] as shown in Fig. 3, the momentum distribution is essentially flat and confined completely to the lower band.

In Fig. 5, the quasimomentum distribution is plotted for two points $\theta=0, \pi / 2$ along the pump path with $R_{\delta}=0.5$ and $R_{\Delta}=2.3$ for a number of interaction strengths, including the hardcore-boson limit $(U / J=\infty)$. The projection onto both the upper $(\alpha=u)$ and the lower band $(\alpha=l)$ of the noninteracting model is shown. The momentum distribution of the physical particles for this path is far from the flat, fermionic distribution, even in the hardcore-boson limit. As the interaction strength approaches $U_{c}$, the momentum distribution becomes increasingly peaked at $k=0$ and develops power-law tails.

We find that the projection onto the upper band is relatively small $\left[n_{u}(k, \theta) \lesssim 0.03\right]$, even far away from the hardcore limit, although it increases as one approaches the central degeneracy.

\section{B. Integrated single-particle Berry curvature}

The expression Eq. (4) for the pumped charge $\Delta Q$ for noninteracting fermions can be interpreted as the accumulated curvature picked up in each filled band over the course of a pump cycle. In the interacting case, one might, by analogy, consider the momentum-weighted single-particle Berry curvature [99], which we define as

$$
\Omega_{\alpha}^{W}(k, \theta)=\Omega_{\alpha}(k, \theta) n_{\alpha}(k, \theta),
$$

with the single-particle Berry curvature and the momentum distribution for each band $\alpha$. The maxima of the singleparticle Berry curvature in $k$-space occur at the band-gap minima where $k a= \pm \pi$; along the pump path they occur at $\theta=m \pi$ for $m \in \mathbb{Z}$, but it still has a significant magnitude throughout the pump cycle. Therefore, charge is pumped at any point during the cycle. Figure 6(a) shows the single-

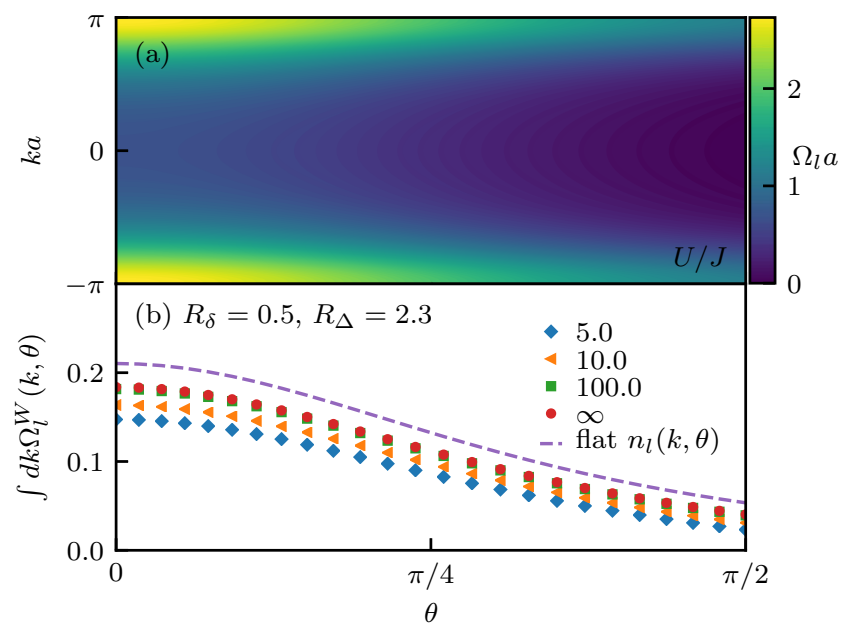

FIG. 6. Single-particle Berry curvature: (a) Berry curvature $\Omega_{l}(k, \theta)$ [Eq. (5)] used for computing the integrated curvature. (b) Partially integrated weighted curvature [defined in Eq. (17)], along the pump cycle for path II. The total integrated charge $\Delta Q_{l}^{\prime} / q$ after one pump cycle is simply the area under these curves between $\theta=0$ and $2 \pi . \Delta Q_{l}^{\prime} / q$ for all paths can be seen in Fig. 7 and are smaller than one.

particle Berry curvature $\Omega_{l}$ for the lower band of path II ( $R_{\delta}=0.5, R_{\Delta}=2.3$ ).

The single-particle picture which motivates Eq. (17) assumes that we simply sum up the contributions from each momentum state to compute the transported charge, which leads to a quantized $\Delta Q_{\alpha}$ when there is a filled band. However, for our model with a finite $U / J$, this will no longer necessarily give a quantized amount for the charge transport due to the inhomogenous occupation in $k$ space.

In analogy to Eq. (4), we can define the integral of the weighted single-particle Berry curvature $\Omega_{\alpha}^{W}$ defined in Eq. (17):

$$
\Delta Q_{\alpha}^{\prime}=q \int_{-\pi / a}^{\pi / a} d k \int_{0}^{2 \pi} d \theta \Omega_{\alpha}^{W}(k, \theta) .
$$

$\Delta Q_{\alpha}^{\prime}$ will, in general, deviate from the exactly quantized $\Delta Q_{\alpha}$ since, in general, $n_{\alpha}(k, \theta)$ is not flat.

The partially integrated weighted curvature is shown in Fig. 6(b) for different points during the pump cycle. The contributions are generally smaller than the corresponding values obtained for a filled band of noninteracting fermions [dashed line in Fig. 6(b)]. Results for $\Delta Q_{\alpha}^{\prime}$ are plotted in Fig. 7 as a function of $U / J$ for the paths shown in Fig. 2 and for the experimental path. For the experimental path, $\Delta Q_{l}^{\prime} \approx q$ even for small interaction strength. The reason is that the bosons remain essentially localized to individual sites during the entire pump cycle. For the elliptical paths that take the system closer to the superfluid region and even for hardcore bosons, $\Delta Q_{l}^{\prime} / q$ can deviate significantly from one. For instance, for path II at $U / J=\infty$, we have $\Delta Q_{l}^{\prime} / q=$ 0.81 . This is consistent with the fact discussed above (see Sec. IV A) that the momentum distribution of hardcore bosons is, in general, not flat for any filling of less than one boson per site. 


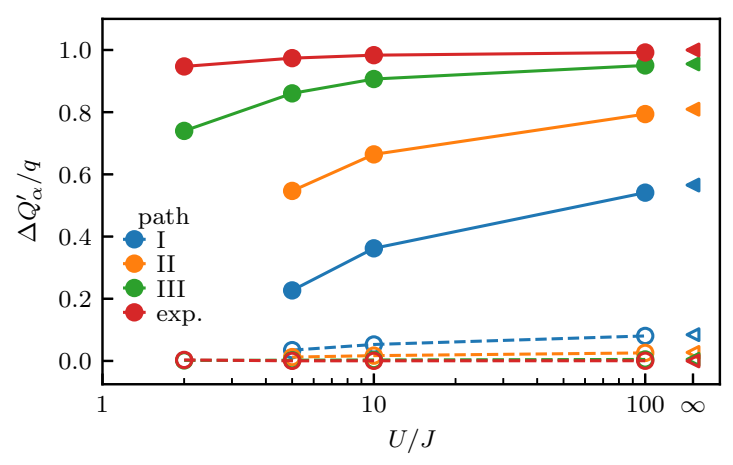

FIG. 7. Breakdown of quantized single-particle invariant: Integral $\Delta Q_{\alpha}^{\prime} / q$ over the weighted single-particle Berry curvature [defined in Eq. (18)] after one pump cycle. We also include the path used in the bosonic charge-pump experiment [55] for comparison, for which the numbers are very close to 1 . In general, though, $\Delta Q_{\alpha}^{\prime}$ is not quantized. Full(empty) symbols indicate $\Delta Q_{\alpha}^{\prime}$ for the lower(upper) band. Triangles indicate the $U / J \rightarrow \infty$ limit.

\section{ASPECTS OF AN EXPERIMENTAL REALIZATION}

Finally, we discuss deviations from ideal conditions relevant to an experimental realization of interacting charge pumps. These aspects are of similar nature as those already found in previous experiments $[55,56]$. In particular, the transport may be affected by imperfect initial state preparation, trap, and finite-size effects. Furthermore, a high measurement accuracy is necessary to resolve the deviations in the transport. However, the main challenge is to perform the interacting charge pump adiabatically with respect to the many-body gap, while keeping technical heating processes sufficiently low. In the hardcore-boson limit, the relevant gap is given by the minimum single-particle band gap [see Eq. (8)], which corresponds to $\Delta E / J=$ 1 for pump path II. When moving away from the hardcore interacting case, the many-body gap reduces rapidly. For example, the minimal many-body gaps for pump path II at two exemplary interaction strengths are $\Delta E / J=$ 0.075 for $U / J=5$ and $\Delta E / J=0.21$ for $U / J=10$. From Fig. 7, we know that for this pump path, significant deviations from the single-particle description exist even in the hardcore-boson limit, which we expect to be observable in future experiments by combining the center-of-mass measurements $[55,56]$ with observations of the momentum distribution $n_{\alpha}(k, \theta)$ using band-mapping techniques [88]. To evaluate the weighted Berry curvature, knowledge about the single-particle Berry curvature $\Omega_{\alpha}(k, \theta)$ is required. This can be derived from the well-known Rice-Mele model. Alternatively, it could be measured independently using statetomography techniques $[42,100]$.

\section{ENTANGLEMENT SPECTRUM}

The entanglement spectrum, defined with respect to a spatial partition of the system into two (semi-infinite) halves, is given by the eigenvalues of the entanglement Hamiltonian $H_{E}$ [80]:

$$
\rho_{L}=e^{-H_{E}}
$$

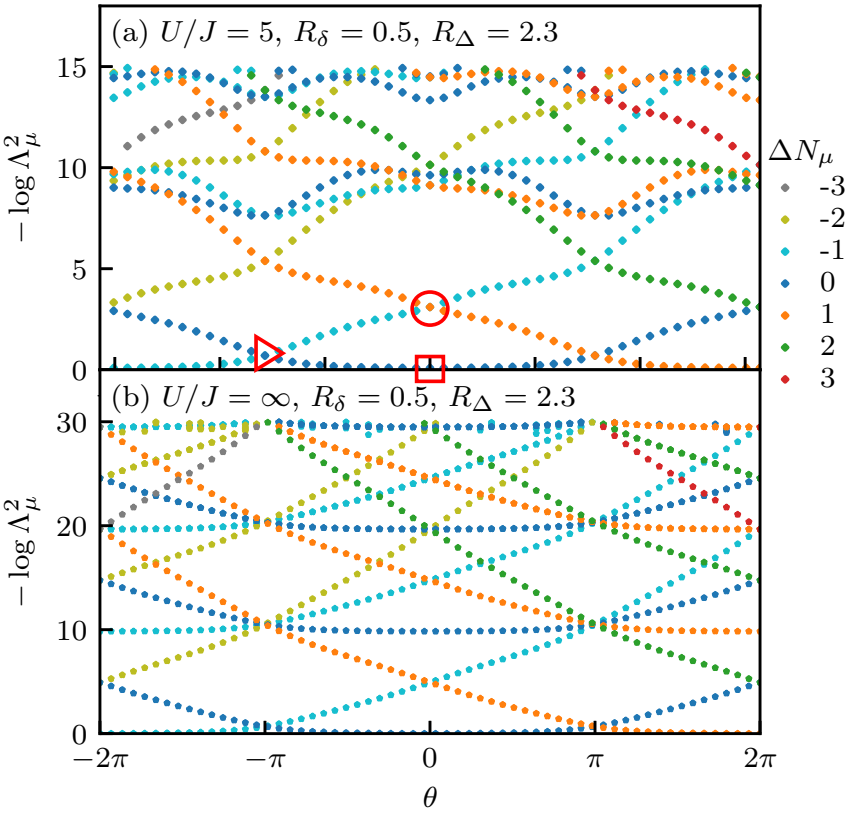

FIG. 8. Entanglement spectrum of the Rice-Mele model: Results for the pump cycle with $R_{\delta}=0.5, R_{\Delta}=2.3$ for (a) $U / J=5$ (from iDMRG) and (b) the $U / J=\infty$ limit, the latter calculated from the free-fermion solution. Note the different scales for the two spectra. The labels $\Delta N_{\mu}$ correspond to the particle imbalance for each Schmidt value. Notice that entanglement eigenvalues in the spectrum wind either up or down or have no winding if $\Delta N_{\mu}=\Delta N_{0}$. After one cycle, the spectrum has the same values, but with all labels increased by 1 , indicating the pumping of a single charge.

$H_{E}$ is unitless and is constrained to be strictly positive by the normalization requirement of the reduced density matrix. The eigenvalues of $H_{E}$ are referred to as entanglement eigenvalues (EEVs).

The flow of the entanglement spectrum is smooth and continuous for a gapped state which evolves under adiabatic perturbations, as is the situation in the charge pump under consideration here. The topological nature of the charge pump is revealed directly in the spectral flow of the entanglement spectrum. Figure 8 shows the entanglement spectrum over the course of one pump cycle $\left(R_{\delta}=0.5, R_{\Delta}=2.3\right)$. Particlenumber conservation allows each EEV to be labeled with a particle imbalance $\Delta N_{\mu}$ across the chosen cut. We plot the EEVs for $-2 \pi<\theta<2 \pi$ and first focus the discussion on the interval $\theta \in[-\pi, \pi)$, which results in a unique lowest EEV $(\mu=0)$ for $\theta \in(-\pi, \pi)$, to which we assign the imbalance $\Delta N_{0}=0$.

Figure 8(a) shows the spectrum for $U / J=5$, computed using iDMRG. Using MPS to represent the ground state throughout the pump cycle makes studying entanglement spectra particularly easy as the eigenvalues $\Lambda_{\mu}$ of the reduced density matrix are computed as part of the iDMRG calculation along with the $\Delta N_{\mu}$. Figure 8(b) shows the corresponding data for $U / J=\infty$, computed from the free-fermion solution (see, e.g., Ref. [101]).

First, note the two symmetric points at $\theta=0$ and at $\theta=$ $-\pi(\pi)$, corresponding to the trivial and nontrivial phases of the SSH model, respectively. This arises from the lattice 
inversion symmetry and imposes a structure on the spectrum. In general, this protected symmetry also requires a fermion parity symmetry [102], but in our model this is automatically satisfied by the $U(1)$ symmetry. The inversion symmetry implies that the spectrum should be invariant under $\Delta N_{\mu} \leftrightarrow$ $-\Delta N_{\mu}$. This requires that EEVs must come in pairs with $\Delta N_{\mu}, \Delta N_{\nu}(\mu \neq \nu)$ such that $\Delta N_{\mu}+\Delta N_{\nu}=N_{0}(\theta)$ or as singlets with $\Delta N_{\mu}=N_{0}(\theta)$, where $N_{0}(\theta)$ is an (integer) constant that can differ for $\theta \in\{-\pi, 0\}$. The existence or absence of the singlet (or, equivalently, if $N_{0}$ is even or odd) distinguishes between the two phases of the SSH model. This relationship between symmetry and entanglement spectrum is well established $[103,104]$.

Furthermore, the spectrum is periodic but the different EEVs can have nontrivial winding over a pump cycle. EEVs from a pair at $\theta=-\pi$ with $\Delta N_{\mu}<0\left(\Delta N_{\mu}>0\right)$ wind up (down) and EEVs with $\Delta N_{\mu}=0$ have trivial winding. After one cycle, the spectrum has the same set of EEVs, but with $\Delta N_{\mu} \rightarrow \Delta N_{\mu}+1$, increased by one [compare the imbalances for $|\theta|>\pi$ to those for $\theta \in(-\pi, \pi)]$.

The increase of labels can be understood by considering the spectral flow between the topologically nontrivial SSH state (at $\theta=-\pi$ ) and the trivial one $(\theta=0)$. At $\theta=-\pi$, there is no singlet but only pairs. To reach the trivial phase at $\theta=0$, pairs containing a state with $\Delta N_{\mu}=0$ must split up, with one state becoming a singlet at $\theta=0$, and the other forming a pair with another EEV from a different pair. This is marked in Fig. 8(a) as squares and circles, respectively. For example, in our choice of $\Delta N_{\mu}$, at $\theta=0$ we have $N_{0}=0$, and the singlets (red square) have $\Delta N_{\mu}=0$. The singlet is connected to pairs at $\theta=-\pi$ of $\Delta N_{\mu}=0,-1$ (red triangle) and $N_{0}=-1$. The EEV with $\Delta N_{\mu}=0$ becomes the singlet and the EEV with $\Delta N_{\mu}=-1$ forms a pair with an EEV with $\Delta N_{\mu}=+1$ (red circle).

At $\theta=\pi$, the EEVs must all form pairs again. The nontrivial winding of the EEVs implies that $N_{0}(\pi) \neq N_{0}(-\pi)$. In our case, $N_{0}(\pi)=1$, and the lowest pair now has $\Delta N_{\mu}=0,1$ as discussed above.

The connection to charge pumping can be made explicit by considering the expression for the many-body polarization in Eq. (16). The shift in $\Delta N_{\mu}$ by some integer over one cycle implies that the polarization changes smoothly and also increases by exactly the shift

$$
\begin{aligned}
P(\theta+2 \pi) & =q a \sum_{\mu} \Lambda_{\mu}^{2}(\theta+2 \pi) \Delta N_{\mu} \\
& =q a \sum_{\mu} \Lambda_{\mu}^{2}(\theta)\left(\Delta N_{\mu}+1\right) \\
& =P(\theta)+q a .
\end{aligned}
$$

Finally, we compare the entanglement spectra at $U / J=5$ to the one at $U / J=\infty$ [see Figs. 8(a) and 8(b)]. As $U / J$ decreases, the EEVs shift to lower values, thus compressing the spectrum and giving more weight to higher particle fluctuations. Moreover, the entanglement spectrum of hardcore bosons exhibits higher degeneracies, inherited from the freefermion case. These degeneracies are broken by the interaction term in Eq. (7) when deviating from $U / J=\infty$. Lowering the interaction strength preferences states with larger fluctuations in the local density (while the average density remains at 1/2), leading to a higher occupation of the states with larger relative $\Delta N_{\mu}$. The topological structure described above, however, is preserved.

\section{SUMMARY}

We showed that topology protects the quantized charge pumping in the interacting bosonic Rice-Mele model in the Mott-insulating regime of one boson per unit cell away from the regime of hardcore bosons. The computation of the quantized pumped charge requires a full many-body calculation via the polarization. We further demonstrated that for interacting bosons with $U_{c} / J<U / J \leqslant \infty$, the single-particle properties of the physical particles never capture the quantized pumping. In the hardcore-boson limit, due to the mapping to free fermions via the Jordan-Wigner transform, a single-particle interpretation is still possible in terms of these fermions. We propose to carry out an experiment in the Mott-insulating regime but with $U / J<\infty$. A measurement of the centerof-mass of the cloud would establish the quantized charge per pump cycle. Measuring the quasimomentum distribution throughout the pump cycle would allow one to demonstrate the breakdown of a single-particle interpretation when combining this information with the known single-particle Berry curvature of the bands.

We also show how the quantized transport is reflected in the structure of the entanglement spectrum and the symmetries of the lattice. The existence of different symmetry-protected topological phases inherited from the SSH model along the pump cycle constrains the degeneracy structure of the entanglement spectrum, enforcing the quantization of the charge transport in the Mott-insulating regime.

\section{ACKNOWLEDGMENTS}

We acknowledge very helpful discussions with I. Bloch, V. Gurarie and F. Pollmann. This work was supported by the Deutsche Forschungsgemeinschaft (DFG, German Research Foundation) under Project No. 277974659 via Research Unit FOR 2414. C.S., M.L., and M.A. additionally acknowledge support from DFG, Project No. 282603579 (DIP), the European Commission (UQUAM Grant No. 5319278), and the Nanosystems Initiative Munich (NIM) Grant No. EXC4. The idea for this project was conceived at KITP, University of Santa Barbara, during the program SYNQUANT16 in discussions with I. Bloch. The hospitality at KITP is gratefully acknowledged. This research was supported in part by the National Science Foundation under Grant No. NSF PHY1748958.
[1] S. Rachel, Rep. Prog. Phys. 81, 116501 (2018).

[2] X.-L. Qi and S.-C. Zhang, Rev. Mod. Phys. 83, 1057 (2011).
[3] M. Z. Hasan and C. L. Kane, Rev. Mod. Phys. 82, 3045 (2010). 
[4] N. R. Cooper, J. Dalibard, and I. B. Spielman, arXiv:1803.00249 [Rev. Mod. Phys. (to be published)].

[5] M. Aidelsburger, S. Nascimbene, and N. Goldman, C. R. Phys. 19, 394 (2018).

[6] V. Galitski and I. Spielman, Nature (London) 494, 49 (2013).

[7] K. L. Hur, L. Henriet, A. Petrescu, K. Plekhanov, G. Roux, and M. Schiro, C. R. Phys. 17, 808 (2016).

[8] T. Ozawa, H. M. Price, A. Amo, N. Goldman, M. Hafezi, L. Lu, M. Rechtsman, D. Schuster, J. Simon, O. Zilberberg, and I. Carusotto, arXiv:1802.04173.

[9] A. Altland and M. R. Zirnbauer, Phys. Rev. B 55, 1142 (1997).

[10] A. P. Schnyder, S. Ryu, A. Furusaki, and A. W. W. Ludwig, Phys. Rev. B 78, 195125 (2008).

[11] A. Rivas, O. Viyuela, and M. A. Martin-Delgado, Phys. Rev. B 88, 155141 (2013).

[12] C.-E. Bardyn, M. A. Baranov, C. V. Kraus, E. Rico, A. İmamoğlu, P. Zoller, and S. Diehl, New J. Phys. 15, 085001 (2013).

[13] D. Linzner, L. Wawer, F. Grusdt, and M. Fleischhauer, Phys. Rev. B 94, 201105 (2016).

[14] J.-H. Zheng and W. Hofstetter, Phys. Rev. B 97, 195434 (2018).

[15] F. Grusdt, Phys. Rev. B 95, 075106 (2017).

[16] C.-E. Bardyn, L. Wawer, A. Altland, M. Fleischhauer, and S. Diehl, Phys. Rev. X 8, 011035 (2018).

[17] M. D. Caio, N. R. Cooper, and M. J. Bhaseen, Phys. Rev. Lett. 115, 236403 (2015).

[18] P. Wang and S. Kehrein, New J. Phys. 18, 053003 (2016).

[19] Y. Hu, P. Zoller, and J. C. Budich, Phys. Rev. Lett. 117, 126803 (2016).

[20] J. C. Budich and M. Heyl, Phys. Rev. B 93, 085416 (2016).

[21] J. H. Wilson, J. C. W. Song, and G. Refael, Phys. Rev. Lett. 117, 235302 (2016).

[22] C. Wang, P. Zhang, X. Chen, J. Yu, and H. Zhai, Phys. Rev. Lett. 118, 185701 (2017).

[23] T. Qin, A. Schnell, K. Sengstock, C. Weitenberg, A. Eckardt, and W. Hofstetter, Phys. Rev. A 98, 033601 (2018).

[24] Z. Wang, X.-L. Qi, and S.-C. Zhang, Phys. Rev. Lett. 105, 256803 (2010).

[25] V. Gurarie, Phys. Rev. B 83, 085426 (2011).

[26] Z. Wang and S.-C. Zhang, Phys. Rev. X 2, 031008 (2012).

[27] Z. Wang and B. Yan, J. Phys.: Condens. Matter 25, 155601 (2013).

[28] H. Shen, W. Wang, and X. Yi, Sci. Rep. 4, 6455 (2014).

[29] Z. Huang and D. P. Arovas, Phys. Rev. Lett. 113, 076407 (2014).

[30] J. C. Budich and S. Diehl, Phys. Rev. B 91, 165140 (2015).

[31] D. J. Thouless, M. Kohmoto, M. P. Nightingale, and M. den Nijs, Phys. Rev. Lett. 49, 405 (1982).

[32] Q. Niu and D. Thouless, J. Phys. A: Math. Gen. 17, 2453 (1984).

[33] C. N. Varney, K. Sun, M. Rigol, and V. Galitski, Phys. Rev. B 84, 241105 (2011).

[34] A. M. Turner, A. Vishwanath, and C. O. Head, Topol. Insul. 6, 293 (2013).

[35] T. Yoshida, R. Peters, S. Fujimoto, and N. Kawakami, Phys. Rev. Lett. 112, 196404 (2014).

[36] M. Aidelsburger, M. Lohse, C. Schweizer, M. Atala, J. T. Barreiro, S. Nascimbène, N. R. Cooper, I. Bloch, and N. Goldman, Nat. Phys. 11, 162 (2015).
[37] Z. Wu, L. Zhang, W. Sun, X.-T. Xu, B.-Z. Wang, S.-C. Ji, Y. Deng, S. Chen, X.-J. Liu, and J.-W. Pan, Science 354, 83 (2016).

[38] L. Asteria, D. T. Tran, T. Ozawa, M. Tarnowski, B. S. Rem, N. Fläschner, K. Sengstock, N. Goldman, and C. Weitenberg, arXiv:1805.11077.

[39] M. Tarnowski, F. N. Ünal, N. Fläschner, B. S. Rem, A. Eckardt, K. Sengstock, and C. Weitenberg, arXiv:1709.01046.

[40] G. Jotzu, M. Messer, R. Desbuquois, M. Lebrat, T. Uehlinger, D. Greif, and T. Esslinger, Nature 515, 237 (2014).

[41] L. Duca, T. Li, M. Reitter, I. Bloch, M. Schleier-Smith, and U. Schneider, Science 347, 288 (2015).

[42] N. Fläschner, B. S. Rem, M. Tarnowski, D. Vogel, D.-S. Lühmann, K. Sengstock, and C. Weitenberg, Science 352, 1091 (2016).

[43] M. Atala, M. Aidelsburger, M. Lohse, J. T. Barreiro, B. Paredes, and I. Bloch, Nat. Phys. 10, 588 (2014).

[44] M. Mancini, G. Pagano, G. Cappellini, L. Livi, M. Rider, J. Catani, C. Sias, P. Zoller, M. Inguscio, M. Dalmonte, and L. Fallani, Science 349, 1510 (2015).

[45] B. K. Stuhl, H.-I. Lu, L. M. Aycock, D. Genkina, and I. B. Spielman, Science 349, 1514 (2015).

[46] M. Atala, M. Aidelsburger, J. T. Barreiro, D. Abanin, T. Kitagawa, E. Demler, and I. Bloch, Nat. Phys. 9, 795 (2013).

[47] E. Meier, F. A. An, and B. Gadway, Nat. Commun. 7, 13986 (2016).

[48] M. Leder, C. Grossert, L. Sitta, M. Genske, A. Rosch, and M. Weitz, Nat. Commun. 7, 13112 (2016).

[49] M. Aidelsburger, M. Atala, M. Lohse, J. T. Barreiro, B Paredes, and I. Bloch, Phys. Rev. Lett. 111, 185301 (2013).

[50] H. Miyake, G. A. Siviloglou, C. J. Kennedy, W. C. Burton, and W. Ketterle, Phys. Rev. Lett. 111, 185302 (2013).

[51] C. J. Kennedy, W. C. Burton, W. C. Chung, and W. Ketterle, Nat. Phys. 11, 859 (2015).

[52] M. E. Tai, A. Lukin, M. Rispoli, R. Schittko, T. Menke, D. Borgnia, P. M. Preiss, F. Grusdt, A. M. Kaufman, and M. Greiner, Nature 546, 519 (2017).

[53] F. A. An, E. J. Meier, and B. Gadway, Sci. Adv. 3, e1602685 (2017).

[54] D. J. Thouless, Phys. Rev. B 27, 6083 (1983).

[55] M. Lohse, C. Schweizer, O. Zilberberg, M. Aidelsburger, and I. Bloch, Nat. Phys. 12, 350 (2016).

[56] S. Nakajima, T. Tomita, S. Taie, T. Ichinose, H. Ozawa, L. Wang, M. Troyer, and Y. Takahashi, Nat. Phys. 12, 296 (2016).

[57] C. Schweizer, M. Lohse, R. Citro, and I. Bloch, Phys. Rev. Lett. 117, 170405 (2016).

[58] H.-I. Lu, M. Schemmer, L. M. Aycock, D. Genkina, S. Sugawa, and I. B. Spielman, Phys. Rev. Lett. 116, 200402 (2016).

[59] Y. E. Kraus, Y. Lahini, Z. Ringel, M. Verbin, and O. Zilberberg, Phys. Rev. Lett. 109, 106402 (2012).

[60] M. Verbin, O. Zilberberg, Y. Lahini, Y. E. Kraus, and Y. Silberberg, Phys. Rev. B 91, 064201 (2015).

[61] M. Lohse, C. Schweizer, H. M. Price, O. Zilberberg, and I. Bloch, Nature 553, 55 (2018).

[62] O. Zilberberg, S. Huang, J. Guglielmon, M. Wang, K. P. Chen, Y. E. Kraus, and M. C. Rechtsman, Nature 553, 59 (2018).

[63] R. Requist and E. Gross, J. Phys. Chem. Lett. 9, 7045 (2018). 
[64] M. Nakagawa, T. Yoshida, R. Peters, and N. Kawakami, Phys. Rev. B 98, 115147 (2018).

[65] E. Berg, M. Levin, and E. Altman, Phys. Rev. Lett. 106, 110405 (2011).

[66] Y. Kuno, K. Shimizu, and I. Ichinose, New J. Phys. 19, 123025 (2017).

[67] Y. Ke, X. Qin, Y. S. Kivshar, and C. Lee, Phys. Rev. A 95, 063630 (2017).

[68] M. P. Zaletel, R. S. Mong, and F. Pollmann, J. Stat. Mech. (2014) P10007.

[69] A. G. Grushin, J. Motruk, M. P. Zaletel, and F. Pollmann, Phys. Rev. B 91, 035136 (2015).

[70] F. Grusdt and M. Höning, Phys. Rev. A 90, 053623 (2014).

[71] R. Resta, Rev. Mod. Phys. 66, 899 (1994).

[72] R. Resta, Phys. Rev. Lett. 80, 1800 (1998).

[73] R. Resta, Ferroelectrics 136, 51 (1992).

[74] R. D. King-Smith and D. Vanderbilt, Phys. Rev. B 47, 1651(R) (1993).

[75] J. Zak, Phys. Rev. Lett. 62, 2747 (1989).

[76] M. J. Rice and E. J. Mele, Phys. Rev. Lett. 49, 1455 (1982).

[77] I. P. McCulloch, arXiv:0804.2509.

[78] G. Vidal, Phys. Rev. Lett. 98, 070201 (2007).

[79] U. Schollwöck, Ann. Phys. (NY) 326, 96 (2011).

[80] H. Li and F. D. M. Haldane, Phys. Rev. Lett. 101, 010504 (2008).

[81] L. Fidkowski, Phys. Rev. Lett. 104, 130502 (2010).

[82] F. Pollmann, A. M. Turner, E. Berg, and M. Oshikawa, Phys. Rev. B 81, 064439 (2010).

[83] X.-L. Qi, H. Katsura, and A. W. W. Ludwig, Phys. Rev. Lett. 108, 196402 (2012).

[84] X. Chen, Z.-C. Gu, and X.-G. Wen, Phys. Rev. B 84, 235128 (2011).

[85] N. Schuch, D. Pérez-Garcia, and I. Cirac, Phys. Rev. B 84, 165139 (2011).
[86] F. Pollmann, E. Berg, A. M. Turner, and M. Oshikawa, Phys. Rev. B 85, 075125 (2012).

[87] M. A. Cazalilla, R. Citro, T. Giamarchi, E. Orignac, and M. Rigol, Rev. Mod. Phys. 83, 1405 (2011).

[88] I. Bloch, J. Dalibard, and W. Zwerger, Rev. Mod. Phys. 80, 885 (2008).

[89] V. G. Rousseau, D. P. Arovas, M. Rigol, F. Hébert, G. G. Batrouni, and R. T. Scalettar, Phys. Rev. B 73, 174516 (2006).

[90] F. Grusdt, M. Höning, and M. Fleischhauer, Phys. Rev. Lett. 110, 260405 (2013).

[91] O. Juergensen and D.-S. Luehmann, New J. Phys. 16, 093023 (2014).

[92] F. Dorfner, Doctoral thesis, LMU Munich, 2016.

[93] S. Sachdev, Quantum Phase Transitions (Cambridge University Press, Cambridge, 2011).

[94] T. D. Kühner and H. Monien, Phys. Rev. B 58, R14741 (1998).

[95] M. Fannes, B. Nachtergaele, and R. F. Werner, Commun. Math. Phys. 144, 443 (1992).

[96] H. F. Song, S. Rachel, C. Flindt, I. Klich, N. Laflorencie, and K. Le Hur, Phys. Rev. B 85, 035409 (2012).

[97] J. Eisert, Model. Simul. 3, 520 (2013).

[98] D. Gangardt and G. Shlyapnikov, New J. Phys. 8, 167 (2006).

[99] T. Neupert, L. Santos, C. Chamon, and C. Mudry, Phys. Rev. Lett. 106, 236804 (2011).

[100] T. Li, L. Duca, M. Reitter, F. Grusdt, E. Demler, M. Endres, M. Schleier-Smith, I. Bloch, and U. Schneider, Science 352, 1094 (2016).

[101] I. Peschel and V. Eisler, J. Phys. A: Math. Theor. 42, 504003 (2009).

[102] D. Sticlet, L. Seabra, F. Pollmann, and J. Cayssol, Phys. Rev. B 89, 115430 (2014).

[103] A. M. Turner, F. Pollmann, and E. Berg, Phys. Rev. B 83, 075102 (2011).

[104] J. Sirker, M. Maiti, N. P. Konstantinidis, and N. Sedlmayr, J. Stat. Mech. (2014) P10032. 